\title{
Age-related differences in the neural basis of the subjective vividness of memories: evidence from multivoxel pattern classification
}

\author{
Marcia K. Johnson ${ }^{1,6}$ • Brice A. Kuhl ${ }^{2}$ - Karen J. Mitchell ${ }^{3}$ • Elizabeth Ankudowich ${ }^{4}$. \\ Kelly A. Durbin ${ }^{5}$
}

Published online: 9 April 2015

(C) Psychonomic Society, Inc. 2015

\begin{abstract}
Although older adults often show reduced episodic memory accuracy, their ratings of the subjective vividness of their memories often equal or even exceed those of young adults. Such findings suggest that young and older adults may differentially access and/or weight different kinds of information in making vividness judgments. We examined this idea using multivoxel pattern classification of fMRI data to measure category representations while participants saw and remembered pictures of objects and scenes. Consistent with our hypothesis, there were age-related differences in how category representations related to the subjective sense of vividness. During remembering, older adults' vividness ratings were more related, relative to young adults', to category representations in prefrontal cortex. In contrast, young adults' vividness ratings were more related, relative to older adults, to category representations in parietal cortex. In addition, category representations were more correlated among posterior regions in young than in older adults, whereas correlations
\end{abstract}

Marcia K. Johnson

marcia.johnson@yale.edu

1 Department of Psychology and Interdepartmental Neuroscience Program, Yale University, New Haven, CT, USA

2 Department of Psychology and Center for Neural Science, New York University, New York, NY, USA

3 Department of Psychology, West Chester University of Pennsylvania, West Chester, PA, USA

4 Integrated Program in Neuroscience, McGill University, Montreal, QC, Canada

5 Department of Psychology, University of Southern California, Los Angeles, CA, USA

6 Department of Psychology, Yale University, P.O. Box 208205, New Haven, CT 06520-8205, USA between PFC and posterior regions did not differ between the 2 groups. Together, these results are consistent with the idea that young and older adults differentially weight different types of information in assessing subjective vividness of their memories.

Keywords Episodic memory $\cdot$ Subjective experience . Vividness $\cdot$ Aging $\cdot$ MVPA

Compared to young adults, older adults often have objectively less accurate event memories (Henkel, Johnson, \& De Leonardis, 1998; Mitchell \& Johnson, 2009; Old \& NavehBenjamin, 2008; Spencer \& Raz, 1995). According to the source monitoring framework (SMF; Johnson, Hashtroudi, \& Lindsay, 1993; Mitchell \& Johnson, 2009), these less accurate memories are thought to arise from poor feature encoding and/or poor binding of features into cohesive representations of specific events (Boywitt, Kuhlmann, \& Meiser, 2012; Chalfonte \& Johnson, 1996; Li, Naveh-Benjamin, \& Lindenberger, 2005; Mitchell, Johnson, Raye, Mather, \& D'Esposito, 2000; Naveh-Benjamin, 2000) and from difficulty monitoring (reviving, evaluating) during remembering (Gallo, 2013; Mitchell et al., 2000, Experiment 2).

One might expect that age-related changes in episodic memory accuracy would be accompanied by similar changes in subjective experience-for example, by the feeling that memories are less vivid. However, the relation between objective and subjective memory is not always so straightforward (e.g., Johnson, 2006). For example, poor memory is not always reflected in omissions but rather sometimes in false memories (source misattributions), and older adults often show a higher rate of source misattributions than young adults (e.g., Henkel et al., 1998; Johnson et al., 1993). Furthermore, older adults are more likely than young adults to assign high 
confidence to, or to say they "Remember," source misattributions (e.g., Dodson, Bawa, \& Krueger, 2007; McCabe, Roediger, McDaniel, \& Balota, 2009; Mitchell, Johnson, \& Mather, 2003; Norman \& Schacter, 1997), indicating that older adults do not necessarily experience objectively inaccurate memory as less vivid memory. Also, older adults often give subjective ratings that are no less than the ratings of young adults to old items they do remember accurately (e.g., McDonough \& Gallo, 2013). Together, such findings suggest that, rather than being the result of a lack of memorial information, some older adults' objectively poor memory accuracy likely results from the use of different, and less than optimally diagnostic, features in making memory judgments, compared to young adults (e.g., Gallo 2013; Mitchell, Ankudowich, Durbin, Greene, \& Johnson, 2013).

Direct evidence for this idea comes from studies using a memory characteristics questionnaire (MCQ) to assess participants' subjective experience (Johnson, Foley, Suengas, \& Raye, 1988). When asked to rate their memories on various qualities such as perceptual, semantic, or emotional detail, older and young adults' ratings either do not differ significantly (Hashtroudi, Johnson, \& Chrosniak, 1990; Henkel et al., 1998; Karpel, Hoyer, \& Toglia, 2001; Lyle, Bloise, \& Johnson, 2006; McGinnis \& Roberts, 1996; Norman \& Schacter, 1997), or older adults give higher ratings than young adults on some features (Comblain, D’Argembeau, \& Van der Linden, 2005; Rubin \& Schulkind, 1997). In one study, young and older adults rated their memories for a number of experimental mini-events (e.g., wrapping a package, having coffee and cookies; Hashtroudi et al., 1990). Analysis of the relations among the MCQ ratings found that compared to young adults, older adults' certainty about the accuracy of their memory was significantly more correlated with ratings of their memory for thoughts and feelings; correlations between certainty and ratings of the clarity of memories were very similar for the two groups (Johnson \& Multhaup, 1992). Consistent findings come from a study by Comblain, D'Argembeau, Van der Linden, and Aldenhoff (2004), who asked participants to report the basis of their "Remember" responses for pictures and found that older adults were more likely than young adults to report emotional detail. Such findings suggest that young and older adults may weight features differently when making memory attributions (see also, e.g., Levine, Svoboda, Hay, Winocur, \& Moscovitch, 2002; May, Rahhal, Berry, \& Leighton, 2005). The present study tested this hypothesis by investigating the neural basis of vividness ratings given by young and older adults during perceiving and remembering.

There is evidence suggesting that when perceiving or remembering, older adults show less activity in brain regions that are responsive to visual stimuli (Cabeza et al., 2004; McDonough, Cervantes, Gray, \& Gallo, 2014; see Spreng, Wojtowicz, \& Grady, 2010, for a meta-analysis). For example, in a recent study, during fMRI scanning, McDonough et al. used word cues to probe participants for the amount of perceptual detail they could remember about complex pictures (i.e., objects and people in contexts) that had previously been presented outside the scanner. Behaviorally, they replicated the frequently found pattern of reduced objective accuracy but similar subjective ratings of amount of detail by older, relative to young, adults. Although older adults showed overall less activity during the rating task in regions that process visual information (parahippocampal gyrus, fusiform gyrus, and precuneus), the two age groups showed similar increases in activity levels in these regions for high-compared to lowdetail items, suggesting that older adults were using visual details to make their ratings. The pattern of fMRI findings is consistent with the common view that remembering involves the reactivation of representations that were encoded previously, and with the idea that older adults reactivate less visual information than do young adults during remembering. Taking the fMRI pattern, together with the similar subjective ratings for young and older adults, McDonough et al. concluded that their results indicate that older adults "recalibrate" their subjective ratings given the overall reduced levels of visual information available. However, findings from this study do not rule out the possibility that young and older adults may be differentially relying on different types of information in making subjective judgments. Specifically, one possibility is that when remembering visual stimuli, older adults differentially rely on representations of semantic or emotional information.

Whereas explicit memory for perceptual details of events is better among young than older adults (Ly, Murray, \& Yassa, 2013; May et al., 2005; Mitchell et al., 2013)_even when implicit measures suggest older adults have encoded perceptual detail (Koutstaal, 2003) — semantic memory is relatively intact among older adults (Balota, Dolan, \& Duchek, 2000). For example, compared to young adults, older adults provide relatively more semantic details in recalling autobiographical events (Levine et al., 2002) and show increased semantically related memory errors (e.g., Balota et al., 1999; Koutstaal et al., 2003; Mitchell et al. 2003). Evidence from fMRI studies indicates that semantic memory errors are associated with activation increases in prefrontal cortex (Garoff-Eaton, Kensinger, \& Schacter, 2007) and that prefrontal activity positively scales with the confidence in semantic errors (Kim \& Cabeza, 2007). Prefrontal cortex may contribute to semantic memory errors by retrieving semantic information from lateral temporal lobe structures (Badre \& Wagner, 2002). Prefrontal cortex may also directly support abstract, conceptual representations that can "fill in" when perceptual details are weak or absent. Indeed, a hallmark of prefrontal cortex is the ability to form conceptual representations that are not tied to sensory or perceptual qualities of stimuli (Miller, Freedman, \& Wallis, 2002; Sreenivasan, Vytlacil, \& D’Esposito, 2014).

Aging is also associated with increased interest in the socio-emotional aspects of events (Carstensen \& Turk- 
Charles, 1994; Scheibe \& Carstensen, 2010), including emotional information during episodic remembering (e.g., Comblain et al., 2004; May et al., 2005). To the extent that older adults are more likely to base their vividness ratings on socio-emotional aspects of a memory than on perceptual details of a memory, this also might be reflected in a reliance on representations in prefrontal cortex. Activity in medial frontal cortex increases during emotional and/or social information processing (Amodio \& Frith, 2006; Etkin, Egner, \& Kalisch, 2011; Kensinger, Allard, \& Krendl, 2014; Lieberman, 2006), and there is evidence that medial prefrontal cortex represents distinct emotional categories (Peelen, Atkinson, \& Vuilleumier, 2010).

Here, we used fMRI to assess age-related differences in the neural basis of subjective ratings of memory vividness. However, rather than testing for univariate activation differences that scale with memory vividness, we used multivoxel pattern analysis (MVPA; see Norman, Polyn, Detre, \& Haxby, 2006; Rissman \& Wagner, 2012, for reviews) to index visual category representations corresponding to perceived/remembered stimuli, and then assessed how the strength of these representations in distinct brain regions related to memory vividness. Prior MVPA-based fMRI studies have found that visual category representations at encoding predict later remembering (Kuhl, Rissman, \& Wagner, 2012) and that patterns of neural activity elicited during encoding are reactivated during remembering (Buchsbaum, Lemire-Rodger, Fang, \& Abdi, 2012; Johnson, McDuff, Rugg, \& Norman, 2009; Johnson \& Johnson, 2014; Kuhl, Rissman, Chun, \& Wagner, 2011; Lee, Kravitz, \& Baker, 2012; Polyn, Natu, Cohen, \& Norman, 2005). Indeed, reactivation at retrieval scales with memory accuracy (Gordon, Rissman, Kiani, \& Wagner, 2013; Kuhl et al., 2011).

Although prior studies have not used MVPA to compare age-related differences in memory vividness, some studies have used MVPA to compare information discriminability in young and older adults. For example, discriminability is lower for older, relative to young, adults when comparing faces versus houses in a passive viewing paradigm (Park, Carp, Hebrank, Park, \& Polk, 2010), and when comparing verbal and spatial working memory maintenance at high but not low working memory loads (Carp, Gmeindl, \& Reuter-Lorenz, 2010; see also Payer et al., 2006). Most relevant to the current study, St-Laurent, Abdi, Bondad, and Buchsbaum (2014) showed participants short video clips (e.g., man crying, roller coaster) intermixed with cues to remember the videos. Each video was presented and cued for recall several times, and MVPA was used to assess the discriminability of the videos during encoding and recall. Young and older adults showed similar levels of stimulus differentiation during perception (greater for young than old only in fusiform gyrus). However, in an analysis of the extent to which stimulus patterns during perception were reactivated during remembering, young adults showed greater stimulus differentiation in several regions, including parietal, temporal, and occipital areas. Interestingly, as found in several studies mentioned above, older adults did not give lower vividness ratings than young adults on recall trials and, in fact, they were more likely than young adults to use the highest vividness rating. However, StLaurent et al. did not report analyses relating vividness ratings to MVPA measures. Thus, we know of no studies to date that use MVPA to assess age-related differences in the neural mechanisms that underlie subjective vividness during perception and remembering. ${ }^{1}$

In the current study, fMRI data were collected as young and older adults encoded and subsequently remembered visual stimuli from two categories (scenes and objects), and multivoxel pattern classification of the fMRI data (a form of MVPA) was used to index the neural discriminability of visual categories. Critically, we also had participants make a single rating of the visual vividness of each item, separately during encoding and remembering. When using MCQ ratings to assess the subjective experience of young and older adults on multiple qualities of events (e.g., perceptual, semantic, emotional), or when using Remember/Know ratings where multiple qualities of events are relevant, age differences may reflect that older adults have greater difficulty shifting attention among the different qualities assessed than do young adults. It is not clear whether young and older adults would differ in subjective experience (and associated brain activity) on any particular characteristic of memories if that were the only feature they had to attend to. That is, with a targeted feature, the relation between neural activity representing any particular feature and subjective vividness might not differ with age. Alternatively, given the well-established finding that older adults have difficulty ignoring irrelevant information (e.g., Hasher \& Zacks, 1988; Mitchell et al., 2013), the vividness ratings when a single feature is targeted may be more influenced by other characteristics of memories for older than for young adults. For example, older adults may be influenced in making perceptual judgments by how they felt about the item, or other things it reminded them of. Hence, of primary interest was the relationship between young and older adults' visual vividness ratings and their MVPA measures during remembering.

\footnotetext{
${ }^{1}$ Unpublished studies by Bloise (2008) comparing subjective ratings using MCQ or General Labeled Magnitude Scales (gLM, a type of scale intended to minimize differences in scale interpretation; Bartoshuk, Duffy, Fast, Green, \& Snyder, 2001) indicated that agerelated dissociations between memory accuracy and subjective ratings are unlikely simply to reflect differences between young and older adults in how they interpret and use MCQ scales. Bloise also found preliminary evidence of differences between young and older adults in the relation between MCQ ratings and activity in brain areas (assessed with univariate analyses). However, her study did not attempt to specifically focus participants' attention on perceptual details during encoding and remembering.
} 
We also assessed the relationship between category information and vividness ratings during encoding, and the relationship between vividness ratings and reactivation (i.e., the extent to which neural patterns activated during remembering were similar to those activated during encoding). The features that support vividness during perception are not necessarily the same as those that support vividness during remembering. Our primary question in this study is not the veridicality of memory (inaccurate memories can be vivid) but rather whether the basis (and hence, the neural correlates) of the subjective experience of vividness during remembering differs between age groups.

Given age-related differences during remembering have been found in a number of brain areas (e.g., Gallo, 2013; Mitchell et al., 2013; St-Laurent et al., 2014), we examined category information in four major brain regions (prefrontal, temporal, parietal, occipital). Specifically, separate pattern classifiers indexed the strength of category information (scene vs. object) within each region based on neural activity during encoding, neural activity during remembering, and the reactivation of encoding activity during remembering. Of central interest were the relations between these neural measures of category information and subjective vividness. According to the SMF (Johnson et al., 1993), agendas affect what information is activated and it is not a foregone conclusion that all information that is active will affect conscious, subjective experience (e.g., Johnson et al., 2009). We anticipated that visual vividness ratings during remembering would differentially relate to category information across brain regions and, most critically, that young and older participants' vividness ratings would be related to category information in different brain regions. We predicted that young adults' vividness ratings during remembering would be relatively more related than older adults' ratings to representations in posterior brain regions, that is, in occipital, temporal, and/or parietal cortex, for example, areas associated with both seeing and imagining scenes (Johnson \& Johnson, 2014) and objects (James, Humphrey, Gati, Menon, \& Goodale, 2000; Konkle \& Oliva, 2012; Newman, Klatzky, Lederman, \& Just, 2005). In contrast, we predicted that older adults' vividness ratings would be more related to representations in prefrontal cortex that potentially reflect abstract, categorical representations (Miller et al., 2002; Sreenivasan et al., 2014) and/or the representation of emotional information (Peelen et al., 2010). We did not make hypotheses about more specific subregions of posterior and anterior cortex for several reasons: (a) the regions showing age differences in univariate fMRI analyses may or may not be the same regions identified in multivariate pattern classification analyses, and hence hypotheses are less clear; (b) a more areageneral analysis involves fewer comparisons, reducing the chances of inflating Type I errors; and (c) looking at larger areas may be more sensitive, especially if more than one subregion in a given brain area shows similar relationships.

\section{Method}

\section{Participants and design}

Young participants ( $n=24,16$ females, $M$ age $=20.8$ years, $S D=2.8$ years; range $=18-29$ years) were college students; older adults $(n=23,14$ females, $M$ age $=71.6$ years, $S D=6.3$ years; range $=61-87$ years) were healthy, independently living adults from surrounding communities. The number of participants (target $n=24$ per age group) was determined in advance based on sample sizes used in the most relevant aging studies (e.g., Carp et al., 2010, $\mathrm{n}_{\mathrm{y}}=18, \mathrm{n}_{\mathrm{o}}=23$; St-Laurent et al., 2014, $\mathrm{n}_{\mathrm{y}}=14, \mathrm{n}_{\mathrm{o}}=14 ;$ McDonough et al., 2014, $\mathrm{n}_{\mathrm{y}}=22$, $\mathrm{n}_{\mathrm{o}}=18$; Park et al., 2010, $\left.\mathrm{n}_{\mathrm{y}}=19, \mathrm{n}_{\mathrm{o}}=19\right){ }^{2}$ Note that our $n \mathrm{~s}$ per group exceeded those in these other published aging studies and hence should yield adequate power to detect differences of interest in our main analyses.

All participants self-reported being in good health, with no history of stroke, serious heart disease, or primary degenerative neurological disorder. Relative to young adults, on a self-report scale from 1 to 5 (where $1=$ excellent $)$, older adults reported better current physical health (i.e., today); $\left(M_{\text {young }}=2.0, M_{\text {older }}=1.3\right)$, $t(45)=3.5, p=.001$, but reports of overall health (past year) did not differ $\left(M_{\text {young }}=1.7, M_{\text {older }}=1.6, p>\right.$ .50). Older adults rated their emotional well-being as better than young adults $\operatorname{did}\left(M_{\text {young }}=1.9, M_{\text {older }}=\right.$ 1.2), for current (today) $t(44)=3.0,{ }^{3} p=.004$; for general (past year) $M_{\text {young }}=2.2, M_{\text {older }}=1.4, t(45)=2.9$, $p=.006$. All participants had normal or corrected to normal vision, and none were taking psychotropic medications. Older adults scored high on the Folstein Mini Mental State Examination $(M=29.3, S D=1.0$; $\max$ possible $=30)$. There was no age-group difference $(p>$ .10) on an abbreviated version of the verbal subscale of the Wechsler Adult Intelligence Scale ( $M_{\text {young }}=21.9$, $S D=5.9, M_{\text {older }}=20.1, S D=6.0 ;$ max possible $=$ 30 ), and older adults had more years of education than did young adults (reported in years, $12=$ high school diploma; $M_{\text {young }}=13.6, S D=2.1, M_{\text {older }}=15.7, S D=$ $2.9 ; t(45)=2.7, p=.01$. All participants were paid. The Human Investigation Committee of Yale University approved the protocol; informed consent was obtained from all participants.

\footnotetext{
${ }^{2}$ Six additional participants were dropped before analyses began: Two older adults (one for excessive motion [29 instances of motion of $9 \mathrm{~mm}$ or more between successive scans], and one whose vision could not be corrected adequately with scanner-safe glasses); four young adults (three for failure to fully engage in the task [e.g., missed $>8 \%$ of the trials], and one who reported engaging in complex, nonvisual strategies during remembering). No other participants were excluded, except from specific analyses, as noted in text (see footnote 5).

${ }^{3}$ One older adult participant did not answer this question.
} 


\section{Stimuli and procedure}

As shown in Fig. 1, participants saw pictures of objects and scenes (See) and later were cued with the pictures' labels to remember the pictures (Think). Picture stimuli were drawn from several sources, including the Internet. They were 60 full-color natural and manmade objects on yellow backgrounds and 60 full-color indoor and outdoor scenes, including landscapes, buildings, and interior rooms (all stimuli were $300 \times 300$ pixels). Across the experiment, each item appeared only once as a picture in a See trial and its label appeared only once in a corresponding Think trial.

Participants were verbally instructed on the tasks outside the scanner and practiced three See-Think cycles using stimuli not used in the scanner. Instructions were clarified as necessary, and participants practiced again if needed. Each See cycle began with a 2 -s cue ("See"; with 1 -s blank before and after), followed by pictures and labels of two objects and two scenes, randomly intermixed and shown for 6-s each (see Fig. 1). Participants were instructed to rate the visual vividness of each item as they looked at it on a scale from 1 (low) to 4 ( high). Each Think cycle began with a 2-s cue ("Think"; with $1 \mathrm{~s}$ blank before and after) followed by each of the four labels corresponding to the just-seen four pictures, for $6 \mathrm{~s}$ each, presented in a different random order. Participants were instructed to "bring to mind, as fully as you can, the picture that had just previously appeared with that label and then rate the visual vividness of the image that you bring to mind, on a scale from 1 (low) to 4 (high)." The instructions further emphasized that participants were to rate the mental image they were able to bring to mind and not simply repeat the rating they had given the picture when they saw it. Participants were told that for both See and Think, vividness refers to the perceived or remembered item's visual characteristics, such as how bright, brilliant, colorful, sharp, distinctive, or dimensional it was. Each stimulus, for both See and Think, was followed by a 6-s intertrial interval (ITI) during which three random numbers (one or two digits each) were shown (750 ms each) and participants were instructed to think "odd" or "even," as appropriate, for each number. This was a relatively easy filler

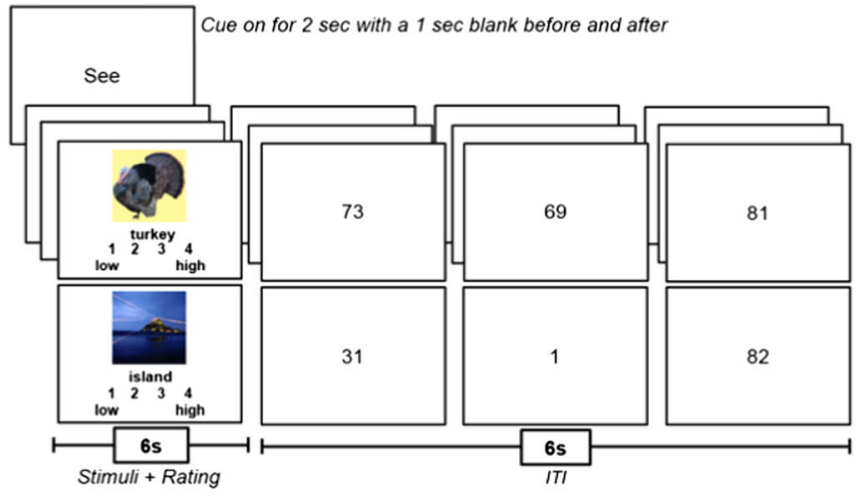

Fig. 1 Procedure and event timeline task used to discourage participants from thinking about the stimuli between trials (e.g., actively trying to maintain them through rehearsal). This filler task, together with the overall time for the See phase of each cycle (48 s) and number of intervening items between Seeing and Thinking about an item (up to 15), makes it unlikely that participants were trying to hold the items active in working memory throughout a SeeThink cycle. Thus, given this timing and the filled intervals between presentation and testing of any particular item, Think vividness ratings should have involved reactivating or retrieving items from long-term memory. In addition, it was emphasized to participants that we were interested in their subjective experience of visual vividness both as they saw pictures and then as they thought about them later. Hence, it was important for them to rate the pictures as they saw them and then later to independently rate them as they remembered them. We used only four items for each See-Think cycle to maximize the likelihood that, even given the need to revive the information from long-term memory, both young and older adults would remember having seen each picture.

Counterbalancing ensured that each ordinal position in each phase contained an object/scene equally often, and that See positions 1 through 4 were queried equally often at Think positions 1 through 4 (to control delay effects). Parallel counterbalancing orders were used for young and older adults. Participants completed six runs of five See-Think cycles each, resulting in a total of 60 trials (360 brain images) per person for each cell of the design.

\section{Imaging details}

After anatomical localizer scans, functional images were acquired on a 3.0T Siemens Trio scanner with a single-shot echoplanar gradient-echo pulse sequence $(\mathrm{TR}=2,000 \mathrm{~ms}$, $\mathrm{TE}=25 \mathrm{~ms}$, flip angle $=80$ degrees, FOV = 240). The 36 oblique axial slices were $3.5 \mathrm{~mm}$ thick with an in-plane resolution of $3.75 \times 3.75 \mathrm{~mm}$ and were aligned with the AC-PC line. Each run began with 12 seconds of blank screen to allow tissue to reach steady state magnetization and was followed by a 1-minute rest interval.

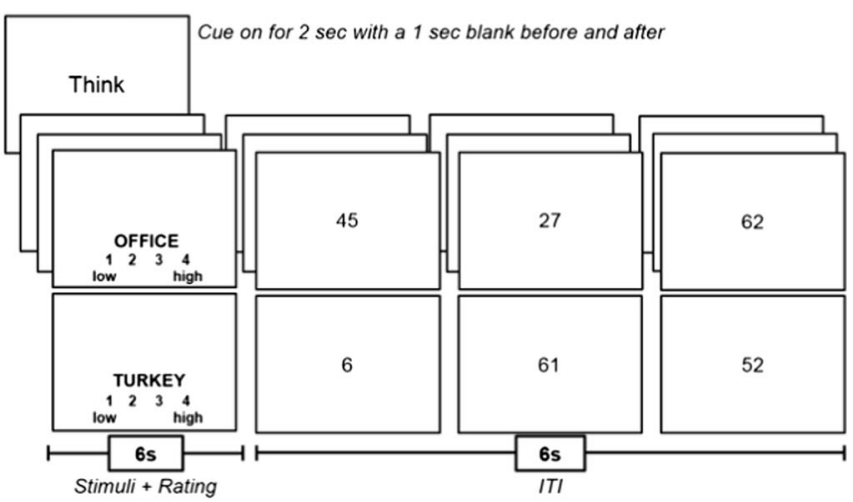




\section{Preprocessing}

Data were motion-corrected using a six parameter automated algorithm (AIR; Woods, Cherry, \& Mazziotta, 1992); trials with motion of $2 \mathrm{~mm}$ or more or rotation of 2 degrees or more between successive scans were removed before analysis (two trials total, each from a different older adult). There was no difference between young and older adults in cumulative motion $(p>.10)$. Although older adults did show significantly more instantaneous (scan-to-scan) motion, measured as total distance, $t(45)=4.497, p<.0001$, the mean sizes of those movements were very small $\left(M_{\text {young }}=.07 \mathrm{~mm}, M_{\text {older }}=.14\right.$ $\mathrm{mm}$ ), minimizing concerns that motion contributed significantly to the results. A 12-parameter AIR algorithm was used to coregister participants' images to an in-house (young) reference brain template. Data were mean-normalized across time and participant and spatially smoothed (3-D, $8 \mathrm{~mm}$ FWHM Gaussian kernel).

Additionally, the data were high-pass filtered $(0.01 \mathrm{~Hz})$, detrended, and $z$ scored across all volumes within each run. A weighted average was applied to the six image volumes corresponding to each trial in the See and the Think phase (three images from the stimulus presentation [the trial itself] and three from the ITI) in order to reduce each trial to a single image pattern. For See, volumes 3 and 4 were equally weighted (.5, .5); for Think, volumes 3, 4, and 5 were averaged, with a stronger weighting on volumes 3 and 4 (.4, .4., .2; Kuhl \& Chun, 2014). A narrower temporal window was used for trials in the See phase than in trials in the Think phase because revival processes tend to be delayed, or more extended in time, relative to encoding processes, as confirmed in a number of independent data sets (e.g., Kuhl et al., 2011). After this temporal compression of the data, a second round of $z$ scoring was performed across all voxels within each (averaged) volume, which resulted in every image volume having a mean response of 0 . Finally, $z$ scoring was also performed for each voxel across all volumes corresponding to the See phase and separately across all volumes corresponding to the Think phase; this removed any mean differences in AMRI activation between the See and Think phases.

\section{Multivoxel pattern analysis}

The fMRI data from the See and Think phases were analyzed using MVPA. In particular, we used pattern classification analyses to decode category representations (object vs. scene) from patterns of neural activity. We applied sparse multinomial logistic regression using the Princeton MVPA toolbox and custom MATLAB code. The penalty parameter was: .05* the total number of voxels within a brain mask. Category classification analyses were performed in three ways: (a) training the classifier on trials in the See phase and testing the classifier on held-out trials in See (See classification), (b) training the classifier on trials in the Think phase and testing the classifier on held-out trials in Think (Think classification), and (c) training the classifier on trials in the See phase and testing the classifier on trials in the Think phase (Reactivation classification). For See and Think classifications, a crossvalidation procedure was used wherein the classifier was always trained using data from five of the six fMRI runs and tested on the run that was held out (e.g., If runs 1, 3, 4, 5, and 6 were used as training, run 2 was used for the test); this was repeated six times (folds) such that every trial contributed to both training and testing, and thus, the same trials served as both held-out and not held-out trials. For reactivation classification, the classifier was trained on trials in the See phase in order to assess reactivation of encoded category information during recall (Think).

As a metric of classifier performance, we report the percentage of trials that were correctly labeled by the classifier (classification accuracy; see Fig. 3). For assessing the relation between classifier performance and vividness ratings, we used a continuous measure of classifier performance as a predictor variable. We used the classifier output, which was a value ranging from 0 to 1 that represented the strength of evidence for category X (and, inversely, for category Y). This output was log transformed before use and is referred to as classifier evidence. Note: classifier evidence is never directly reported below; it was only used as a predictor variable (see Fig. 5).

Pattern classification analyses were separately conducted within four broad regions of interest (ROIs): prefrontal cortex (PFC), temporal lobe (TEMP), parietal cortex (PAR), and occipital cortex (OCC). ROIs were in standard MNI brain space and were generated using the Anatomical Automatic Labeling (AAL) atlas (Tzourio-Mazoyer, et al., 2002). For PFC, the mask consisted of the union of the AAL masks corresponding to inferior frontal gyrus, middle frontal gyrus, superior frontal gyrus, medial superior frontal gyrus, anterior cingulate cortex, and medial orbitofrontal gyrus. The TEMP mask consisted of the union of masks corresponding to temporal pole; inferior, middle, and superior temporal gyri; hippocampus; parahippocampal gyrus; and fusiform gyrus. The PAR mask corresponded to the union of angular and supramarginal gyri, superior parietal lobule, precuneus, posterior cingulate, and retrosplenial cortex. The OCC mask corresponded to the union of masks corresponding to inferior, middle, and superior occipital gyri as well as lingual gyrus, calcarine sulcus, and cuneus. All masks were bilateral and were edited such that they did not contain overlapping voxels.

\section{Results}

\section{Vividness ratings}

The distributions of vividness ratings were quite similar for young and older adults; the most notable difference was that 
older adults used the highest rating (4) more often than young adults for objects in both the See and Think phase. Mean vividness ratings are shown in Fig. 2. An age (young, older) $\times$ category (object, scene) $\times$ processing $($ See, Think) ANOVA showed a main effect of age, $F(1,45)=4.07, p=.05$, partial $\eta^{2}$ $=.08$, with older adults giving higher vividness ratings overall $(M=2.93)$ than did young adults $(M=2.70)$. There was also a main effect of process, $F(1,45)=23.70, p<.0001$, partial $\eta^{2}=$ .35 , with items given higher vividness ratings during See $(M=$ $2.88)$ than Think $(M=2.74)$. There was no difference overall between objects and scenes $\left(p>.10\right.$, partial $\left.\eta^{2}<.01\right)$. There was, however, a category $\times$ process interaction, $F(1,45)=$ $8.20, p<.01$, partial $\eta^{2}=.15$, due to a larger difference between See and Think for scenes $(M \mathrm{~s}=2.92,2.72)$ for See and Think, respectively, $t(46)=6.17, p<.001$, than objects $(M \mathrm{~s}=$ $2.84,2.76$ ) for See and Think, respectively, $t(46)=1.91, p=$ .06 . An age $\times$ category interaction, $F(1,45)=8.18, p<.01$, partial $\eta^{2}=.15$, indicated that the difference between young $(M=2.85)$ and older $(M=2.78)$ adults was negligible for scenes $(p>.10)$, but older adults $(M=3.07)$ gave significantly higher ratings to objects than did young adults $(M=2.54, p<$ $.01)$. The interaction of age $\times$ process was marginally significant, $\mathrm{F}(1,45)=3.59, p=.07$, partial $\eta^{2}=.07$, with the difference between young and older adults' vividness ratings greater for Think $\left(M_{\mathrm{y}}=2.60, M_{0}=2.88, p<.05\right)$ than See $\left(M_{\mathrm{y}}\right.$ $\left.=2.79, M_{\mathrm{o}}=2.97, p>.10\right)$. The three-way interaction of age $\times$ category $\times$ process was not significant, $F(1,45)=2.09, p=$ .16 , partial $\eta^{2}=.04$.

We also computed the correlation between See and Think ratings of the same items, expecting that pictures that seem more vivid as they are perceived have qualities that will generate a sense of vividness when they are remembered. Indeed, we found strong correlations in both groups, and a significantly greater correlation in older $(M=.77)$ than in young $(M=$ $.60), t(44)=3.60, p=.001$, adults. Whether this high correlation is due to properties of the pictures themselves (some pictures simply seem more vivid regardless of whether they

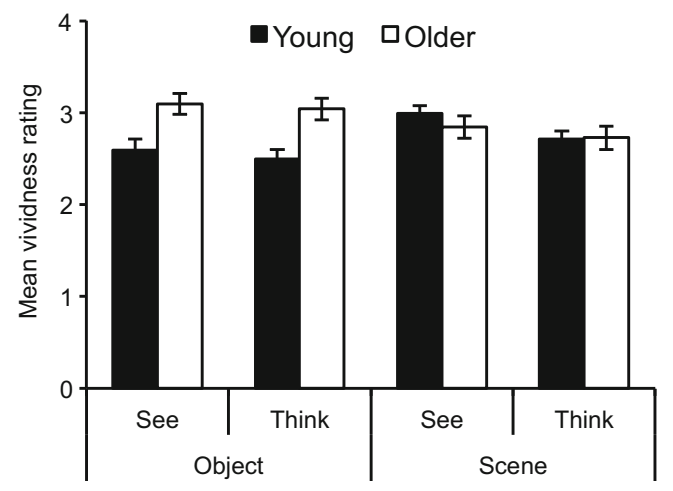

Fig. 2 Mean vividness ratings as a function of Age, Process (See, Think), and Category (object, scene). The main effect of Age and the Age $\times$ Process interaction were each significant $(p s<.05)$. Error bars represent standard error of the mean are being encoded or recalled) or instead reflects an encoding process affecting later retrieval (more vivid encoding causes more vivid subsequent remembering) is difficult to tease apart. This issue is orthogonal to our key questions, and will not be discussed further.

\section{Classification accuracy}

Given two classification categories (object, scene), chance classification accuracy is $50 \%$. Classification accuracy for See and Think (see Fig. 3a, b), as well as reactivation accuracy (see Fig. 4a), were above chance in all regions examined for both groups $(p s<.01)$, with the exception of
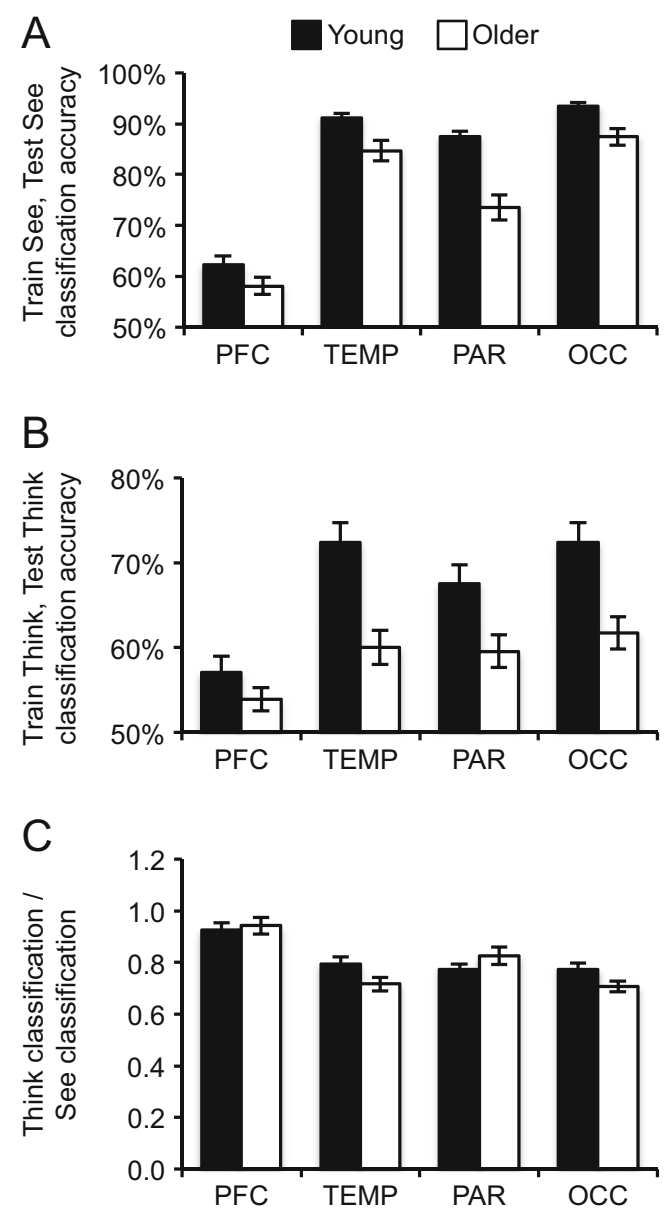

Fig. 3 Classification accuracy for See and Think trials. (a) Category (scene vs. object) classification accuracy for See trials, from classifier trained on See trials. The main effect of Age and the Age $\times$ ROI interaction were each significant $(p s<.05)$. (b) Category classification accuracy for Think trials, from classifier trained on Think trials. The main effect of Age and the Age $\times$ ROI interaction were each significant $(p s<$ .05 ). (c) Category classification accuracy for Think trials as a proportion of classification accuracy on See trials. The Age $\times$ ROI interaction was significant $(p<.05)$. PFC: prefrontal cortex ROI; TEMP: temporal lobe ROI; PAR: parietal lobe ROI; OCC: occipital lobe ROI (ROIs were bilateral; see Method for details). Error bars represent standard error of the mean 

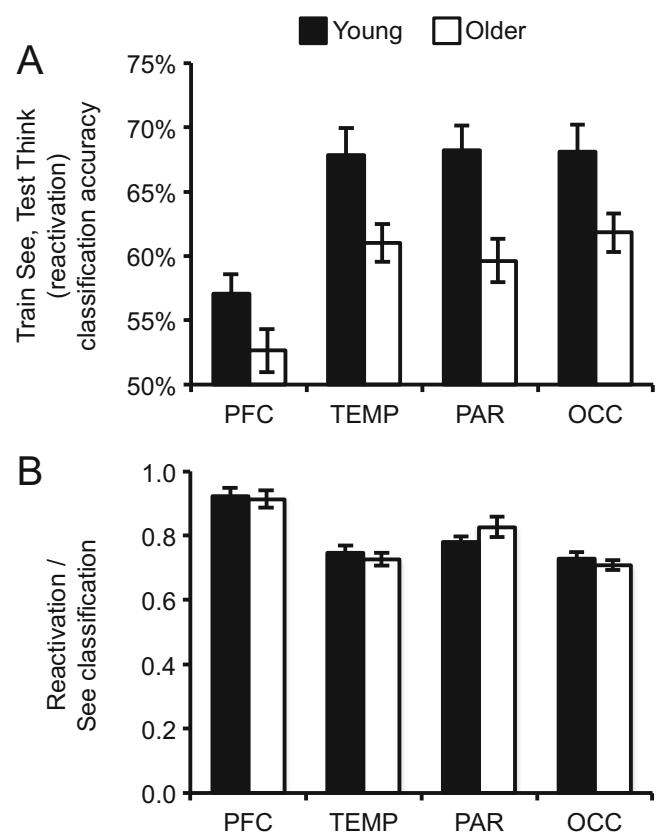

Fig. 4 Classification accuracy based on reactivation of See-phase activity during Think phase. (a) Category classification accuracy for Think trials, from classifier trained on See trials. The main effect of Age was significant $(p<.05)$. (b) Reactivation accuracy (as shown in a) as a function of See classification accuracy. PFC: prefrontal cortex ROI; TEMP: temporal lobe ROI; PAR: parietal lobe ROI; OCC: occipital lobe ROI (ROIs were bilateral; see Method for details). Error bars represent standard error of the mean

older adults' accuracy in PFC in the reactivation analysis (see Fig. $4 \mathrm{a} ; p=.13)$.

\section{Classification of category information during See} trials We first assessed performance of a category classifier (objects vs. scenes) that was trained and tested on trials in the See phase (see Fig. 3a). Classification performance was reflected in mean classification accuracy: the percentage of trials (for each participant and each ROI) that were correctly classified. An ANOVA with factors of age (young, older) and ROI (PFC, TEMP, PAR, OCC) revealed robust main effects of age, $F(1,45)=16.44, p<.001$, partial $\eta^{2}=.27$, and ROI, $F(3$, $135)=334.92, p<.001$, partial $\eta^{2}=.88$ as well as a significant age $\times$ ROI interaction, $F(3,135)=8.10, p<.001$, partial $\eta^{2}=$ .15. As can be seen in Fig. 3a, category classification was lower overall for older adults, but this age-related difference was greater in PAR than in PFC, TEMP, or OCC $(p s<.005)$.

\section{Classification of category information during Think} trials We next assessed performance of a category classifier that was trained and tested using trials in the Think phase (see Fig. 3b). There were main effects of age, $F(1,45)=11.61, p=$ .001 , partial $\eta^{2}=.21$, and ROI, $F(3,135)=42.11, p<.001$, partial $\eta^{2}=.48$ and a significant age $\times$ ROI interaction, $F(3$, $135)=6.06, p=.001$, partial $\eta^{2}=.12$. As can be seen in Fig. 3b, category classification was lower overall for older adults, and the age-related difference was greater in TEMP and OCC than PFC $(p s<.005)$ and marginally greater in PAR than PFC $(p<.07)$.

Thus, category classification was lower for older adults on trials in both the See and Think phase. In order to assess whether the age-related differences on trials in the Think phase were any greater than were those in the See phase, we computed the ratio of Think-phase to See-phase classifier accuracy (Think accuracy/See accuracy; see Fig. 3c). There was no main effect of age $\left(F<1\right.$, partial $\left.\eta^{2}=.02\right)$, but there was a significant main effect of ROI, $F(3,135)=36.15, p<.001$, partial $\eta^{2}=.45$, qualified by a significant age $\times$ ROI interaction, $F(3,135)=4.86, p<.005$, partial $\eta^{2}=.10$. The interaction reflects the fact that the relative strength of Think vs. See classification was lower for older than for young participants in TEMP and OCC $(p s<.05)$, but the age groups did not differ significantly in PFC or PAR ( $p$ s > .10). In short, if we consider See-phase classification accuracy as a baseline for category discriminability within each age group, older adults exhibited a relatively greater drop during the Think phase than did young adults from this baseline in TEMP and OCC. The next analysis addresses whether this category discriminability profile necessarily reflects a greater deficit in reactivation in temporal and occipital cortex in older than young adults.

\section{Reactivation of category information during Think trials}

Training and testing a classifier based on Think-phase data alone addresses the degree to which thoughts of objects and scenes are associated with discriminable activity patterns, but not whether activity present during perception (See phase) is reactivated during remembering (Think phase). To assess this, we trained a classifier to discriminate category information based on See-phase data and then tested it on Think-phase data (see Fig. 4a). Classification accuracy was markedly lower for older than for young adults, $F(1,45)=8.93, p<.005$, partial $\eta^{2}=.17$. Classification accuracy also varied across the ROIs, $F(3,135)=43.27, p<.001$, partial $\eta^{2}=.49$, with PFC classification accuracy lower than each of the other three regions $(p s<.001)$, which did not differ significantly from each other $(p s>.26)$. There was no age $\times$ ROI interaction, $F(3,135)=1.37, p=.26$, partial $\eta^{2}=.03$. Hence, relative to young adults, older adults showed lower levels but a similar profile of reactivation across the four ROIs examined here. When reactivation classification success was expressed as a proportion of See classification success (see Fig. 4b) to take into account differences in initial perception, neither the main effect of age $\left(F<1\right.$; partial $\left.\eta^{2}<.01\right)$ nor age $\times$ ROI interaction $\left(F<1\right.$; partial $\left.\eta^{2}=.04\right)$ were significant. The main effect of ROI was significant, $F(3,135)=52.65, p<.001$, partial $\eta^{2}=$ .54 , reflecting $\mathrm{PFC}>\mathrm{PAR}>\mathrm{TEMP}(p \mathrm{~s}<.001)=\mathrm{OCC}(p=$ $.12)$. Thus, while reactivation was lower in older than in young adults in absolute terms (see Fig. 4a), reactivation 
relative to initial category discriminability was similar between young and older adults in all brain regions (see Fig. 4b).

To summarize the classification accuracy findings, older adults had less differentiated representations of category information during encoding (i.e., during See trials) and this agerelated encoding deficit presumably contributed to less differentiated representations during retrieval (i.e., during Think trials).

\section{Subjective vividness}

We turn next to our main question of how the strength of classifier-based category information relates to the vividness ratings that young and older adults made during See and Think. We applied participant- and ROIspecific linear regression models in which classifier evidence (see Method) was used as an independent (predictor) variable and vividness ratings were the dependent (predicted) variable. ${ }^{4}$ Coefficients (beta values) from the regression model were then entered into second-level random effects analyses ( $t$ tests, ANOVAs). Positive beta values reflect a positive, linear relationship between classifier evidence and vividness; negative beta values reflect a negative linear relationship; beta values close to 0 reflect no relationship. Importantly, generating participant-specific regression coefficients "corrects" for across-participant differences in the use of the vividness rating scale that might arise from different response criteria. For example, if Participant A distributed most/ all of their responses across ratings 1-3, and Participant B distributed most/all responses across ratings 2-4, combining data across participants based on the absolute ratings would be problematic, whereas a regression model can detect a similar linear relationship (if one exists) in these two participants.

Classifier evidence during See trials as a predictor of See vividness We tested for relationships between classifierbased evidence for category information during See trials and vividness ratings made by participants during $\mathrm{See}^{5}$ (see Fig. 5a). An ANOVA with factors of age and ROI revealed a marginal effect of age (Young $<$ Old), $F(1,44)=3.77, p=.06$, partial $\eta^{2}=.08$; no main effect of ROI; $F(3,132)=1.43, p=$ .24 , partial $\eta^{2}=.03$; and a significant age $\times$ ROI interaction, $\mathrm{F}(3,132)=4.69, p<.005$, partial $\eta^{2}=.10$. The interaction reflects the fact that in OCC and TEMP the relationship

\footnotetext{
${ }^{4}$ Classifier evidence was $z$ scored across all trials within each category class. Trials for which no response was made were excluded.

${ }^{5}$ One older adult was excluded from this analysis because the participant responded with highest vividness (4) for every trial in the See phase.
}
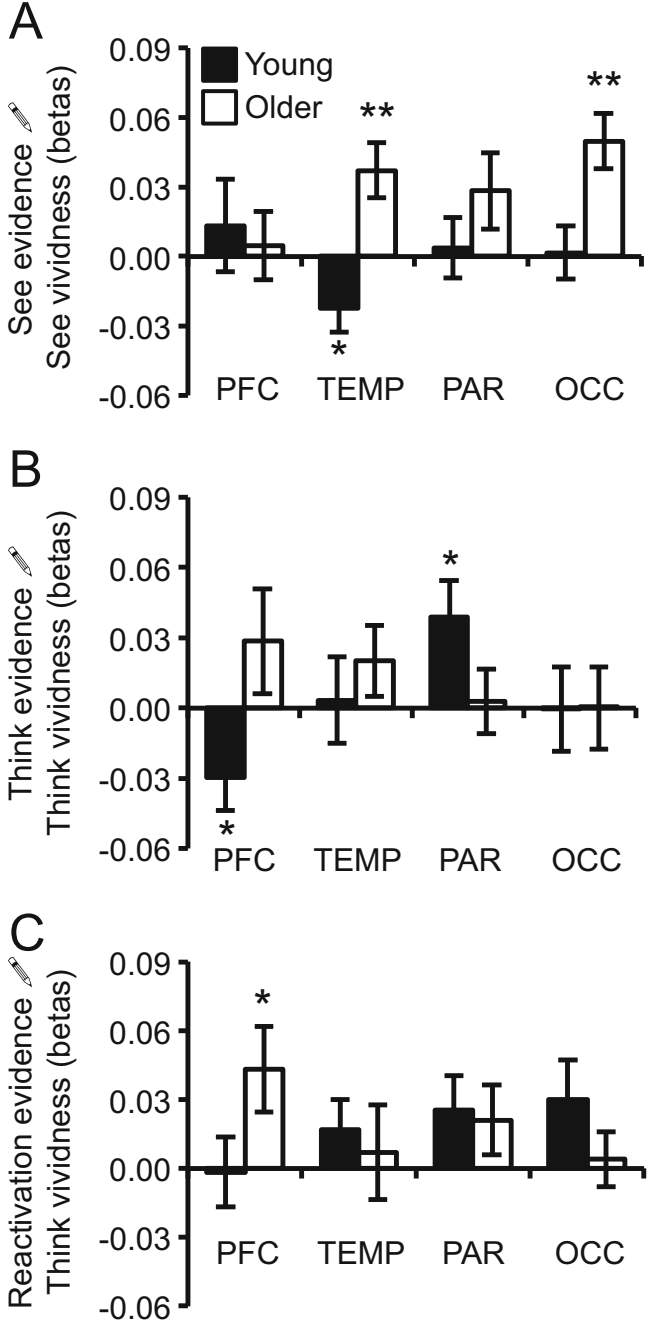

Fig. 5 Relationship between classifier evidence and vividness ratings. (a) Mean beta values from linear regression analyses that predicted See vividness ratings from See classifier evidence. (b) Mean beta values from linear regression analysis that predicted Think vividness ratings from Think classifier evidence. (c) Mean beta values from linear regression analysis that predicted Think vividness ratings from Reactivation evidence. PFC: prefrontal cortex ROI; TEMP: temporal lobe ROI; PAR: parietal lobe ROI; OCC: occipital lobe ROI (ROIs were bilateral; see Method for details). Error bars represent standard error of the mean. * $=$ significantly different from 0 , without correction for multiple comparisons; $* *=$ significantly different from 0 , with correction for multiple comparisons. For $\mathbf{a}, \mathbf{b}$, and $\mathbf{c}$, the Age $\times \mathrm{ROI}$ interactions were significant (see text for details).

between category evidence and vividness ratings was significantly stronger (more positive) in older than in young adults $(p s<.005)$, but this was not the case in PFC or PAR ( $p s>.10)$. Data from subregions within each of the four broad masks are shown in Appendix 1.

Classifier evidence during Think trials as a predictor of Think vividness We also tested for relationships between Think-phase classifier evidence (from the classifier trained and tested on trials in the Think phase) and vividness ratings 
made during Think phase (see Fig. 5b). The main effect of age was not significant $\left(F<1\right.$; partial $\left.\eta^{2}=.01\right)$ and the main effect of ROI was not significant, $F(3,132)=1.17, p=.32$, partial $\eta 2=.03$, but there was a significant age $\times$ ROI interaction, $F(3,132)=4.25, p<.01$, partial $\eta^{2}=.09$. This interaction was largely driven by PFC and PAR. As is clear from Fig. 5b, in PFC there was a more positive relationship between vividness and classifier evidence for older than for young adults $(p<.05$, Cohen's $d=.64$ ), whereas an opposite trend was observed in PAR, with a more positive relationship between vividness and classifier evidence for young than older adults $(p=.09$, Cohen's $d=.50$ ). Although the PAR age difference was marginal by a two-tailed test, it was derived from a significant and predicted interaction and had a "medium" Cohen's $d$ value; thus, we regard this result as consistent with our hypothesis regarding age differences in anterior/posterior brain regions related to vividness during remembering. Data from subregions within each of the four broad masks are shown in Appendix 2.

\section{Relationship between reactivation evidence and subjective vividness on Think trials}

We also tested whether vividness ratings during the Think phase were related to the strength with which See-phase activity patterns were reactivated during the Think phase (see Fig. 5 c). For this analysis, we used classifier evidence for reactivation (classifier training $=$ See, testing $=$ Think). Note that for this analysis we did not use reactivation evidence that was "corrected" for See-phase classification accuracy because the linear regression analyses were applied within-participants and therefore did not depend on overall reactivation magnitude but instead on the linear relationship between reactivation and vividness. Neither the main effects of age nor ROI were significant $\left(F_{\mathbf{s}}<1\right)$, but the age $\times$ ROI interaction was significant, $F(3,135)=2.83, p<.05$, partial $\eta^{2}=.06$. This interaction was driven by a relatively stronger relationship between category evidence and vividness ratings in older adults than young adults in PFC ( $p=.07$, Cohen's $d=.55)$ and opposite, but nonsignificant, age-related differences in the other regions $(p s>.2)$. Data from subregions within each of the four broad masks are shown in Appendix 3.

To summarize the relation between vividness ratings and classifier evidence during remembering, we observed a predicted age $\times$ region interaction in both the analysis of Think data (train Think, test Think) and the analysis of reactivation data (train See, test Think). Across both analyses, the interactions were qualitatively similar: older adults' vividness ratings, compared to young adults' ratings, tended to be more positively related to PFC representations, whereas for young adults, vividness ratings were relatively more related to representations in posterior regions - this was particularly evident when considering PAR effects in the analysis of Think data.

\section{Cross-region correlations in category evidence}

The preceding analyses indicate that relative to young adults, older adults' vividness ratings were more dependent on representations in prefrontal cortex, whereas young adults' vividness ratings were relatively more dependent on representations in posterior regions (PAR, in particular). As a final, exploratory analysis, we compared the similarity of information across brain regions (Kuhl et al., 2012) for each of the two age groups. This allowed us to assess, for example, the coupling between PFC and other regions within older adults and the coupling among posterior regions for young adults. Separate correlations were computed for each measure (See-phase evidence, Think-phase evidence, and Reactivation evidence) by correlating classifier evidence across the relevant trials for each pair of brain regions (e.g., PFC-TEMP). Correlations were separately computed for each participant, and resulting correlation coefficients were $z$ transformed and were then compared via $t$ tests (see Fig. 6). For each age group and each measure (See-phase evidence, Think-phase evidence, Reactivation evidence), correlations between each pair of regions were significantly above chance (mean $z \mathrm{~s}>.18, p \mathrm{~s}<.001$ ). Of critical interest, however, was whether the strength of correlations differed between the age groups. Indeed, ANOVAs with factors of age and region pairing (i.e., the six possible combinations of regions) revealed significant interactions when considering See evidence, $F(5,225)=11.79, p<.001$, partial $\eta^{2}=.21$; Think evidence, $F(5,225)=4.88, p<.001$; partial $\eta^{2}=.10$; and Reactivation evidence, $F(5,225)=8.45$, $p<.001$, partial $\eta^{2}=.16$. Each of these interactions was driven by relatively greater correlations among posterior regions (i.e., TEMP-PAR, PAR-OCC, and TEMP-OCC) for young than for older participants ( $p \mathrm{~s}<.05$ for all comparisons except Think evidence for PAR-OCC), and an absence of age-related differences in the strengths of correlations that involved PFC (PFC-TEMP, PFC-PAR, and PFCOCC; all $p \mathrm{~s}>.2$ ). Thus, for older adults, the relationship between PFC and posterior regions was relatively "preserved," but relationships among posterior regions were almost uniformly weaker compared to young adults. Although these data do not directly relate to vividness ratings, they underscore that age-related differences in perception/remembering may be reflected in the patterns of coupling between regions. More directly relating inter-region coupling to subjective experiences of vividness would be an interesting avenue for future research.

\section{Discussion}

Behaviorally, when instructed to rate visual vividness, older adults' ratings during remembering were as high as those of 


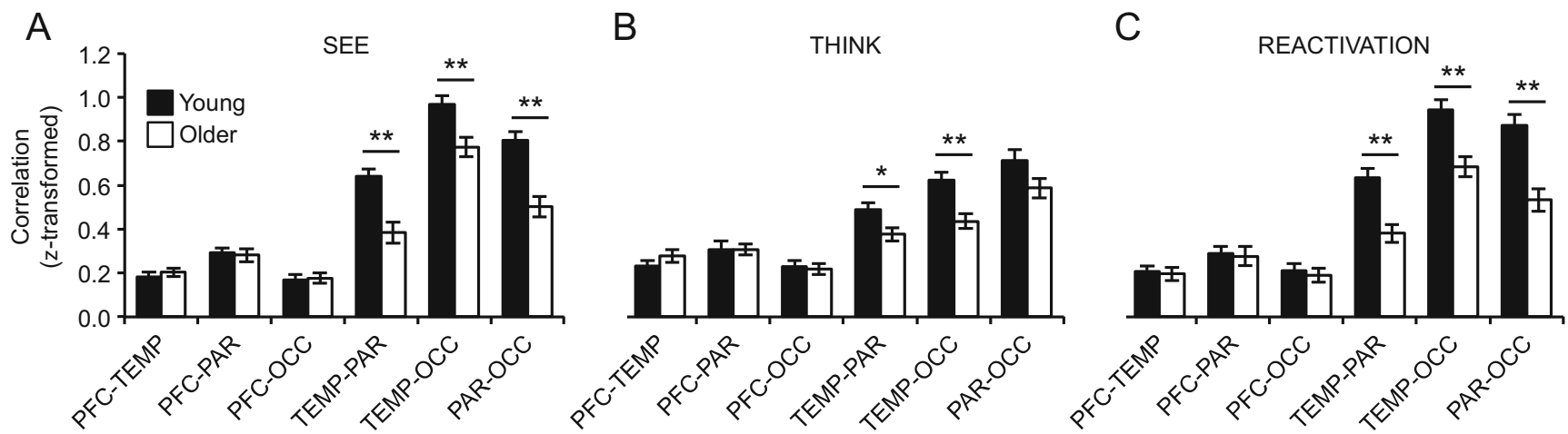

Fig. 6 Age-related differences in the correlations of classifier evidence between brain regions for (a) See-phase evidence, (b) Think-phase evidence, and (c) Reactivation evidence. For each age group, correlations between each pair of regions for each measure (See, Think, Reactivation evidence) were significantly above chance (see text for details). Additionally, the Age $\times$ Region pairing interaction was significant for each measure ( $p$ s $<.001$; see text for details). PFC: prefrontal cortex ROI; TEMP: temporal lobe ROI; PAR: parietal lobe ROI; OCC: occipital lobe ROI (ROIs were bilateral; see Method for details). * = significant effect of age, without correction for multiple comparisons; $* *$ = significant effect of age, with correction for multiple comparisons young adults for scenes and even higher for objects. These results are consistent with previous findings that older adults tend to give higher subjective ratings than one might expect based on objective evidence that their memories for event details are less accurate (e.g., Comblain et al., 2005; Henkel et al., 1998; McDonough et al., 2014; StLaurent et al., 2014). We hypothesized that young and older adults' vividness ratings of their mental experience might be based on different types of features (or on a different weighting of features). In particular, we predicted that during remembering, young adults would be relatively more likely to base their vividness ratings on visual information processed in posterior brain regions (i.e., occipital, temporal, and/or parietal cortex) whereas older adults would be relatively more likely to base vividness ratings on more conceptual/semantic or socio-emotional representations that are supported by prefrontal cortex. Our findings provided support for this prediction. During remembering, the relationship between vividness ratings and classifier evidence in PFC was more positive for older than young adults; in contrast, young adults' vividness ratings were more strongly related to posterior representations (PAR, in particular). The following is a more detailed discussion of the results of this study.

Consistent with previous findings that seeing and thinking about visual information are distributed across a wide range of brain areas (e.g., Buchsbaum et al., 2012; Kuhl, Johnson, \& Chun, 2013; Kuhl \& Chun, 2014; Polyn et al., 2005; St-Laurent et al., 2014), we found significant category classification accuracy for both age groups during encoding and remembering in prefrontal, temporal, parietal, and occipital cortex. While category information was markedly weaker, overall, in older adults, age-related "deficits" were especially apparent in parietal cortex during the See phase (see
Fig. 3A) and in temporal and occipital cortex during the Think phase (see Fig. 3b).

In considering the functional roles of the regions showing age-related differences during encoding and remembering, and how that might be reflected in differences in category classification, one possibility is that parietal cortex contributes to feature binding during or immediately after encoding (Shimamura, 2011), which contributes to category-level representations picked up by our pattern classifier during the See phase. That is, the age-related difference in classification accuracy in parietal cortex during the See phase may reflect the well-established age-related deficit in representing or binding feature combinations (Boywitt et al., 2012; Chalfonte \& Johnson, 1996; Li et al., 2005; Mitchell et al., 2000; Naveh-Benjamin, 2000). The relatively weaker Think-phase category representations in temporal and occipital regions for older adults may then reflect this binding deficit - that is, because of a parietal-related binding deficit, features represented in temporal cortex are less likely to cue features represented in occipital cortex and/or vice versa. This would be consistent with prior evidence for age-related decreases in activity in occipitotemporal regions during recall (e.g., McDonough et al., 2014). The fact that age-related "deficits" in category representations across brain regions may look different depending on whether perceptual processing (See trials) or reflective processing (Think trials) is being assessed highlights the importance of dissociating perceptual and reflective processes for understanding cognition and disruptions in cognition (e.g., Chun \& Johnson, 2011; Lee et al., 2012; Johnson \& Johnson, 2009; Mitchell, Johnson, Higgins, \& Johnson, 2010).

The reactivation analysis (see Fig. 4a) was generally consistent with classification accuracy observed for the Think- 
phase analysis (train Think, test Think; see Fig. 3b) in that older adults' classification accuracy was markedly lower across all regions (see also St-Laurent et al., 2014). However, the ratio of reactivation to See phase classification accuracy (Fig. 4b) was similar for the two age groups, suggesting that older adults "retained" about the same proportion of category information as young adults. This is in contrast to St-Laurent et al., who found age-related reactivation deficits even after controlling for pattern discriminability during encoding. There are many differences between the two studies in procedure and materials. For example, in our study, participants saw and remembered each scene and object once only, and in the St-Laurent et al. study, participants saw and remembered videos multiple times. The differences in procedures suggest a number of future directions to explore. In the meantime, our data indicate that under some circumstances, older adults may not differ from young adults in the proportion of encoding experience activated again during remembering; nevertheless, any reduction in information discriminability during encoding would be expected to be reflected (if not magnified) during remembering.

Our main question was whether there are age-related differences in the information that drives subjective vividness ratings of perceptual and reflective experience. According to the SMF, the fact that information is activated does not necessarily mean that it is used (Mitchell et al., 2013). Similarly, the fact that a pattern classifier can decode information about a remembered stimulus from activity in a given brain region does not necessarily mean that participants use that information in an agenda-relevant way (McDuff, Frankel, \& Norman, 2009; Kuhl et al., 2013). Here, we assessed the relation between classifier evidence and vividness ratings, which provided the critical test of agenda-relevant use of neural category representations. During the See phase, the vividness ratings of older adults were significantly related to classifier evidence in OCC and TEMP regions, suggesting that their subjective sense of vividness during perception was related to visual characteristics processed by these regions. Interestingly, for young adults, no a priori ROI showed a significant correlation with vividness ratings during the See phase. This may reflect more variability in the individual features from different ROIs driving vividness ratings from trial to trial in young adults during perception, or that vividness ratings are more related to combinations of features across ROI regions during perception. In any event, for both young and older adults, vividness ratings during perception (see Fig. 5a) were not a simple function of classifier evidence (see Fig. 3a).

During the Think phase, the relation between classifier evidence and vividness ratings differed for the two age groups as a function of brain region (i.e., there was an age $x$ region interaction in the relationship between classifier evidence and vividness ratings). This was true when considering the analysis in which we trained and tested the pattern classifier on trials in the Think phase (see Fig. $5 b$ ) and in the reactivation analysis (train See, test Think; see Fig. 5c). For both analyses, compared to young adults, older adults' vividness ratings during Think trials were more strongly related to category evidence in PFC, consistent with our prediction that, during remembering, older adults may rely on information other than visual/perceptual details. This result is particularly striking considering that category information in posterior regions (OCC and TEMP) strongly predicted older adults' vividness ratings during See trials (see Fig. 5a). This difference in the regions that contributed to older adults' vividness ratings from See to Think was reflected in a significant interaction between phase and region, $\mathrm{F}(3,63)=3.37, p<.05$, (See [Fig. 5a] vs. Think reactivation analysis [Fig. $5 \mathrm{c}])$. Hence, when perceptual information is available (See phase), older adults readily make use of this information to guide vividness ratings. During recall (Think phase), however, either deficits in encoding and/or retrieving perceptual features (e.g., McDonough et al., 2014; Mitchell et al., 2013) and/or increased reflective attention toward other features (e.g., semantic, affective; e.g., Comblain et al., 2004; Johnson \& Multhaup, 1992; Levine et al., 2002; May et al., 2005; Mitchell et al., 2003, 2013) may shift older adults toward making vividness judgments by accessing or more heavily weighting information represented in PFC.

For young adults, on the other hand, vividness ratings during the Think phase were best predicted by category information in parietal cortex (see Fig. 5b). The relationship between vividness ratings and parietal classifier performance for young adults was significant when considering Think evidence (see Fig. 5b) and qualitatively similar though nonsignificant when considering Reactivation evidence. However, across both analyses, and across parietal subregions (see Appendices 2 and 3), there were qualitatively similar, positive relationships between classifier performance and vividness ratings. It may at first seem surprising that young adults' vividness ratings were not related to classifier evidence in OCC or TEMP regions, given evidence that these regions process visual features of objects and scenes (e.g., Schwarzlose, Swisher, Dang, \& Banisher, 2008), especially since young adults showed substantial classifier evidence in OCC and TEMP during See trials (see Fig. 3a). However, parietal cortex is also involved in scene and object processing (e.g., Johnson \& Johnson, 2014; Vingerhoets, 2014), and is argued to be 
involved in binding features during encoding and remembering (Shimamura, 2011) or representing multifeatured episodic information retrieved from memory (Vilberg \& Rugg, 2008; see also, Wagner, Shannon, Kahn, \& Buckner, 2005). Furthermore, parietal lobe damage is associated with reduced confidence in retrieved memories (Simons, Peers, Mazuz, Berryhill, \& Olson, 2010). Finally, and especially relevant to the present findings, recent studies using MVPA methods similar to those employed here have shown that activity patterns in young adults' lateral parietal cortex robustly reflect retrieved information that is agenda-relevant (Kuhl et al., 2013) and also reflect detailed information (i.e., exemplar-specific information) about retrieved stimuli during successful remembering (Kuhl \& Chun, 2014). In sum, compared to older adults, young adults evidenced (a) relatively better classifier accuracy in PAR during encoding, (b) a relatively stronger relation between PAR classifier performance and vividness ratings during remembering, and (c) stronger correlations among posterior regions, including PAR, for See, Think, and Reactivation evidence. Collectively, these findings are all consistent with the idea that, relative to older adults, young adults perception/ remembering relied more heavily on representations in parietal cortex. These findings add to a growing literature implicating parietal cortex in episodic remembering and provide new evidence relating parietal representations (in young adults) to the subjective vividness of memories.

Although we have focused on the possibility that, relative to young adults, older adults are influenced more by semantic and/or affective information represented in PFC in making vividness ratings, there are likely differences between objects and scenes in the processes they evoke. For example, because scenes tend to be more complex than objects, they likely engage more cognitive operations (e.g., shifts in attention to different details). Assuming cognitive operations generate records (Johnson \& Raye, 1981), these records could contribute to category discrimination between objects and scenes, and PFC activity is associated with records of cognitive processes (e.g., Dobbins, Foley, Schacter, \& Wagner, 2002; Mitchell et al., 2008; Simons, Owen, Fletcher, \& Burgess, 2005). Although classification could certainly occur on the basis of records of which or how many cognitive operations were engaged (e.g., shifts in attention, number of rehearsals), it seems unlikely that vividness ratings would be associated with which or how many cognitive operations were engaged rather than the outcomes of such processes (e.g., the representation of perceptual or semantic information). A related possibility is suggested by the many lines of evidence for the interaction between frontal and parietal cortex in both perceptual and reflective attention (e.g., Chun \& Johnson, 2011). If young adults relatively automatically bind and/or represent integrated feature information in parietal cortex but older adults, because of a binding deficit, are more likely to engage frontal cortex to refresh or rehearse individual features, this would account for vividness ratings associated with information in parietal cortex in young adults and prefrontal cortex in older adults. Another possibility is that older adults have more difficulty than young adults recovering perceptual information and/or monitoring the information recovered and this difficulty engaged prefrontal activity (e.g., the PFC activity reflects repeated retrieval attempts). However, it is not obvious that increasing processing difficulty per se would generate the subjective experience of greater vividness.

Further studies using more specific manipulations of stimuli and orienting tasks and targeting subregions within the broad ROIs used here will be necessary to more completely characterize the features and/or processes that are differentially contributing to the subjective experience of vivid remembering in young and older adults. Likewise, it seems important to investigate how the relation between vividness ratings and brain activity would look at longer delays and/or with more intervening items, as is more typical of long-term memory studies. Although we should generally expect both similarities and differences in regional brain activity at long and short delays (e.g., Cabeza, Dolcos, Graham, \& Nyberg, 2002), of interest would be the outcome of MVPA comparisons, and the interaction with age. For example, with a longer delay and/or more intervening items, for both groups the availability of perceptual details might decrease more than conceptual/ semantic attributes. Thus, with a greater delay younger adults might show a shift toward relying on PFC-dependent representations, which would make their data look more like older adults at the short delay.

Finally, comparing across analyses (Figs. 3a with $5 \mathrm{a} ; 3 \mathrm{~b}$ with $5 \mathrm{~b}$, and $4 \mathrm{a}$ with $5 \mathrm{c}$ ) highlights an important point that is consistent with predictions from the Source Monitoring Framework (Johnson et al., 1993): Age-related differences in the vividness of memories may arise as much (or perhaps even more) from relative differences in what information is reflectively attended and how it is weighted as from how much information is activated (Mitchell et al., 2013). While additional studies will be required to more fully characterize the relation between activation patterns during perception and remembering, reactivation patterns, and the subjective sense of remembering, the current findings illustrate that there is not a one-to-one correspondence between memory activation/ reactivation and memory vividness and that apparent paradoxes between age-related changes in subjective vs. objective measures may be clarified by considering the specific neural information that gives rise to these behavioral measures.

Acknowledgments This research was supported by National Institute on Aging grant R37AG009253 and National Institute of Mental Health grant R01MH092953. The authors declare no conflict of interest, financial or personal. We thank Carol Raye for discussions contributing to the development of this line of research and the Yale Magnetic Resonance Research Center technologists for assistance with fMRI data collection. 


\section{Appendix 1}

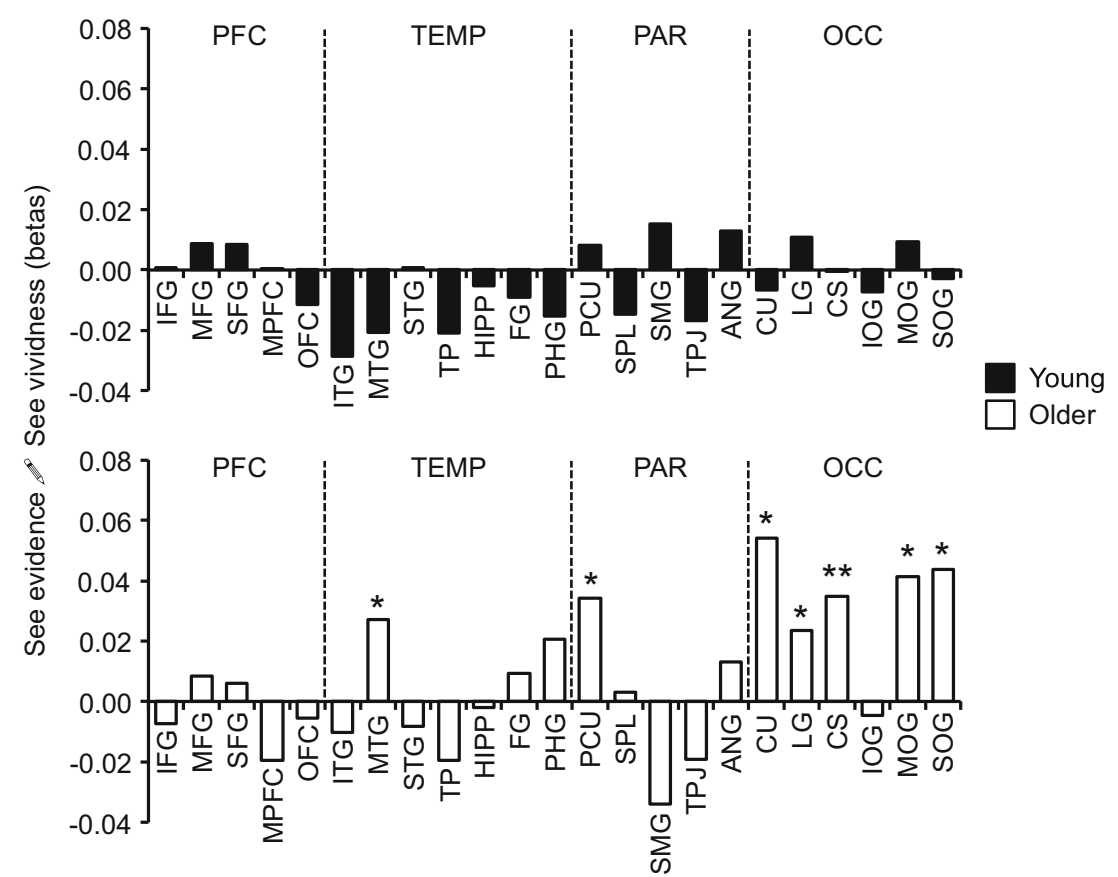

Fig. 7 Relationship between See-phase evidence and See vividness ratings by subregion. $*=$ significantly different from 0 , uncorrected; $* *=$ significantly different from 0 , corrected for multiple comparisons. IFG: inferior frontal gyrus; $\mathrm{MFG}=$ middle frontal gyrus; $\mathrm{SFG}=$ superior frontal gyrus; $\mathrm{MPFC}=$ medial prefrontal cortex; $\mathrm{OFC}=$ orbitofrontal cortex; ITG = inferior temporal gyrus; $\mathrm{MTG}=$ middle temporal gyrus; $\mathrm{STG}=$ superior temporal gyrus; $\mathrm{TP}=$ temporal pole; HIPP $=$ hippocampus; $\mathrm{FG}=$ fusiform gyrus; $\mathrm{PHG}=$ parahippocampal gyrus; $\mathrm{PCU}=$ precuneus; SPL $=$ superior parietal lobule; SMG = supramarginal gyrus; TPJ = temporoparietal junction; $\mathrm{ANG}=$ angular gyrus; $\mathrm{CU}=$ cuneus; $\mathrm{LG}=$ lingual gyrus; $\mathrm{CS}=$ calcarine sulcus; IOG = inferior occipital gyrus; $\mathrm{MOG}=$ middle occipital gyrus; $\mathrm{SOG}$ $=$ superior occipital gyrus 


\section{Appendix 2}

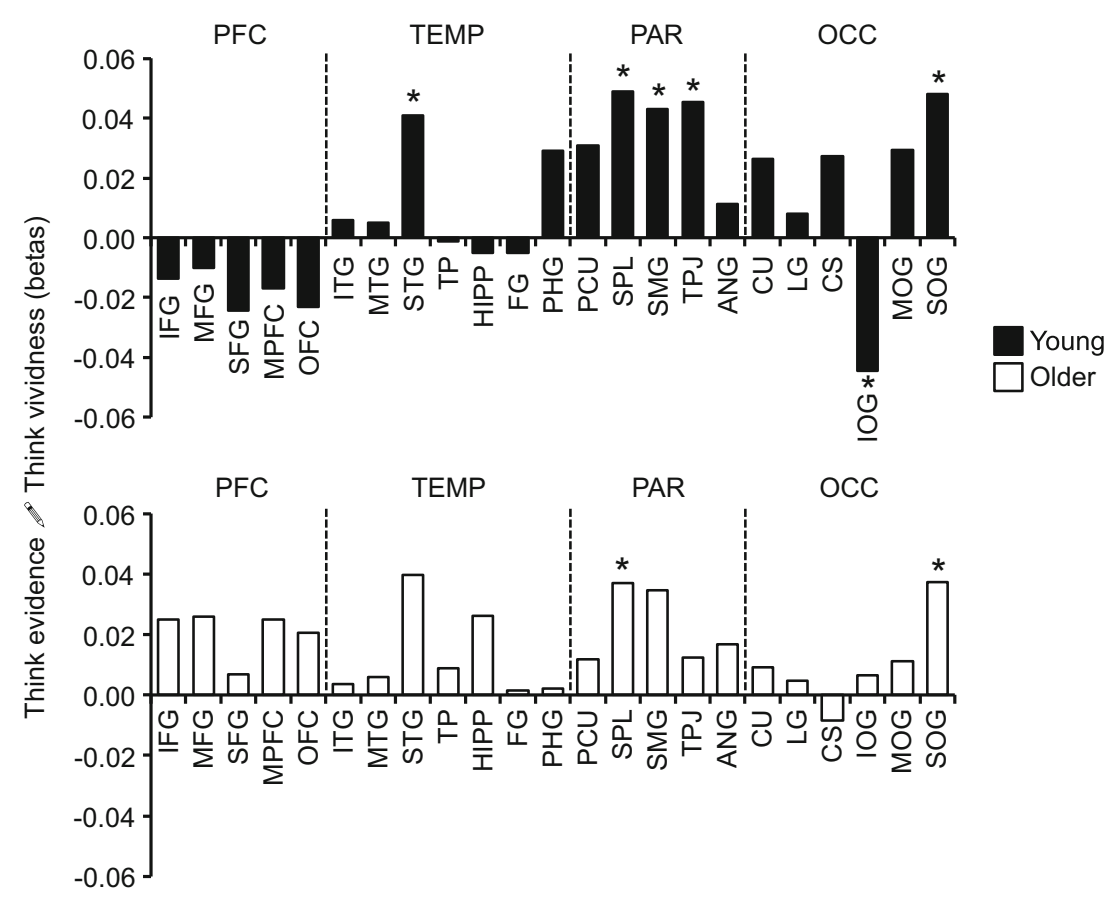

Fig. 8 Relationship between Think-phase evidence and Think vividness ratings by subregion. $*=$ significantly different from 0 , uncorrected. IFG: inferior frontal gyrus; $\mathrm{MFG}=$ middle frontal gyrus; $\mathrm{SFG}=$ superior frontal gyrus; $\mathrm{MPFC}=$ medial prefrontal cortex; OFC $=$ orbitofrontal cortex; $\mathrm{ITG}=$ inferior temporal gyrus; $\mathrm{MTG}=$ middle temporal gyrus; $\mathrm{STG}=$ superior temporal gyrus; $\mathrm{TP}=$ temporal pole; $\mathrm{HIPP}=$ hippocampus; $\mathrm{FG}=$ fusiform gyrus; $\mathrm{PHG}=$ parahippocampal gyrus; $\mathrm{PCU}=$ precuneus; $\mathrm{SPL}=$ superior parietal lobule; $\mathrm{SMG}=$ supramarginal gyrus; $\mathrm{TPJ}=$ temporoparietal junction; $\mathrm{ANG}=$ angular gyrus; $\mathrm{CU}=$ cuneus; 333333LG = lingual gyrus; $\mathrm{CS}=$ calcarine sulcus; $\mathrm{IOG}=$ inferior occipital gyrus; $\mathrm{MOG}=$ middle occipital gyrus; $\mathrm{SOG}=$ superior occipital gyrus 


\section{Appendix 3}

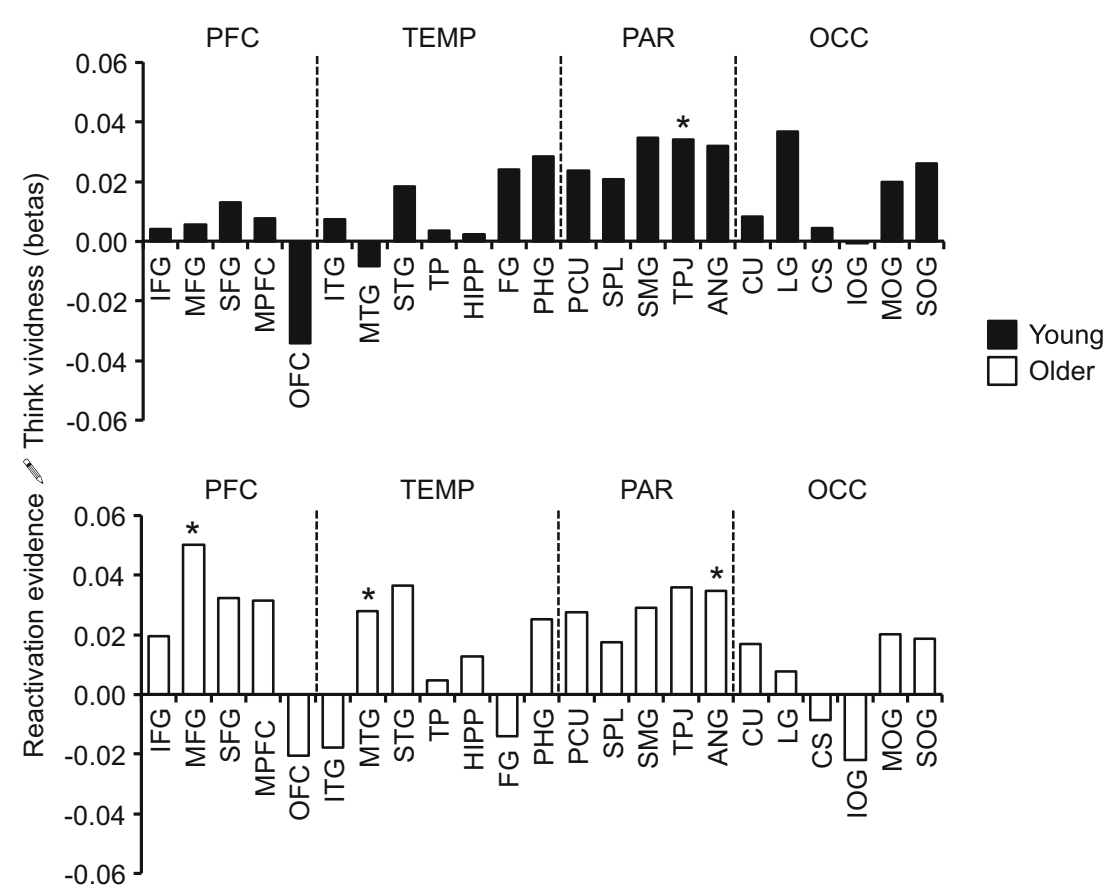

Fig. 9 Relationship between Reactivation evidence and Think vividness ratings by subregion. $*=$ significantly different from 0 . IFG: inferior frontal gyrus; $\mathrm{MFG}=$ middle frontal gyrus; $\mathrm{SFG}=$ superior frontal gyrus; $\mathrm{MPFC}=$ medial prefrontal cortex; $\mathrm{OFC}=$ orbitofrontal cortex; ITG = inferior temporal gyrus; MTG = middle temporal gyrus; STG = superior temporal gyrus; $\mathrm{TP}=$ temporal pole; $\mathrm{HIPP}=$ hippocampus; $\mathrm{FG}=$

\section{References}

Amodio, D. M., \& Frith, C. D. (2006). Meeting of minds: The medial frontal cortex and social cognition. Nature Reviews Neuroscience, 7 , 268-277.

Badre, D., \& Wagner, A. D. (2002). Semantic retrieval, mnemonic control, and prefrontal cortex. Behavioral and Cognitive Neuroscience Reviews, 1, 206-218.

Balota, D. A., Cortese, M. J., Duchek, J. M., Adams, D., Roediger, H. L., McDermott, K. B., \& Yerys, B. E. (1999). Veridical and false memory in healthy older adults and in dementia of the Alzheimer's type. Cognitive Neuropsychology, 16, 361-384.

Balota, D. A., Dolan, P. O., \& Duchek, J. M. (2000). Memory changes in healthy young and older adults. In E. Tulving \& F.I.M. Craik (Eds.), The Oxford handbook of memory (pp. 395-410). New York, NY: Oxford University Press.

Bartoshuk, L. M., Duffy, V. B., Fast, K., Green, B. G., \& Snyder, D. J. (2001). The General Labeled Magnitude Scale provides valid measures of genetic variation in taste and may be a universal psychophysical ruler. Appetite, 37, 126.

Bloise, S. M. (2008). Aging and the subjective experience of remembering. New Haven, CT: Yale University. Unpublished doctoral dissertation.

Boywitt, C. D., Kuhlmann, B. G., \& Meiser, T. (2012). The role of source memory in older adults' recollective experience. Psychology and Aging, 27, 484-497. fusiform gyrus; $\mathrm{PHG}=$ parahippocampal gyrus; $\mathrm{PCU}=$ precuneus; $\mathrm{SPL}=$ superior parietal lobule; $\mathrm{SMG}=$ supramarginal gyrus; $\mathrm{TPJ}=$ temporoparietal junction; $\mathrm{ANG}=$ angular gyrus; $\mathrm{CU}=$ cuneus; $\mathrm{LG}=$ lingual gyrus; $\mathrm{CS}=$ calcarine sulcus; $\mathrm{IOG}=$ inferior occipital gyrus; $\mathrm{MOG}=$ middle occipital gyrus; $\mathrm{SOG}=$ superior occipital gyrus

Buchsbaum, B. R., Lemire-Rodger, S., Fang, C., \& Abdi, H. (2012). The neural basis of vivid memory is patterned on perception. Journal of Cognitive Neuroscience, 24, 1867-1883.

Cabeza, R., Daselaar, S. M., Dolcos, F., Prince, S. E., Budde, M., \& Nyberg, L. (2004). Task-independent and task-specific age effects on brain activity during working memory, visual attention and episodic retrieval. Cerebral Cortex, 14, 364-375.

Cabeza, R., Dolcos, F., Graham, R., \& Nyberg, L. (2002). Similarities and differences in the neural correlates of episodic memory retrieval and working memory. NeuroImage, 16(2), 317-330.

Carp, J., Gmeindl, L., \& Reuter-Lorenz, P. A. (2010). Age differences in the neural representation of working memory revealed by multi-voxel pattern analysis. Frontiers in Human Neuroscience, 4, 217. doi:10.3389/fnhum.2010.00217

Carstensen, L. L., \& Turk-Charles, S. (1994). The salience of emotion across the adult life span. Psychology and Aging, 9, 259-264.

Chalfonte, B. L., \& Johnson, M. K. (1996). Feature memory and binding in young and older adults. Memory \& Cognition, 24, 403-416.

Chun, M. M., \& Johnson, M. K. (2011). Memory: Enduring traces of perceptual and reflective attention. Neuron, 72, 520-535.

Comblain, C., D'Argembeau, A., Van der Linden, M., \& Aldenhoff, L. (2004). The effect of ageing on the recollection of emotional and neutral pictures. Memory, 12, 673-684.

Comblain, C., D’Argembeau, A., \& Van der Linden, M. (2005). Phenomenal characteristics of autobiographical memories for emotional and neutral events in older and younger adults. Experimental Aging Research, 31, 173-189. 
Dobbins, I. G., Foley, H., Schacter, D. L., \& Wagner, A. D. (2002). Executive control during episodic retrieval: multiple prefrontal processes subserve source memory. Neuron, 35(5), 989-996.

Dodson, C. S., Bawa, S., \& Krueger, L. E. (2007). Aging, metamemory, and high-confidence errors: A misrecollection account. Psychology and Aging, 22, 122-133.

Etkin, A., Egner, T., \& Kalisch, R. (2011). Trends in Cognitive Sciences, $15,85-93$.

Gallo, D. A. (2013). Retrieval expectations affect false recollection: Insights from a criterial recollection task. Current Directions in Psychological Science, 22, 316-323.

Garoff-Eaton, R. J., Kensinger, E. A., \& Schacter, D. L. (2007). The neural correlates of conceptual and perceptual false recognition. Learning \& Memory, 14, 684-692.

Gordon, A. M., Rissman, J., Kiani, R., \& Wagner, A. D. (2013). Cortical reinstatement mediates the relationship between content-specific encoding activity and subsequent recollection decisions. Cerebral Cortex. doi:10.1093/cercor/bht194. Advance online publication.

Hasher, L., \& Zacks, R. T. (1988). Working memory, comprehension, and aging: A review and a new view. In G. H. Bower (Ed.), The psychology of learning and motivation (Vol. 22, pp. 193-225). New York, NY: Academic Press.

Hashtroudi, S., Johnson, M. K., \& Chrosniak, L. D. (1990). Aging and qualitative characteristics of memories for perceived and imagined complex events. Psychology and Aging, 5, 119-126.

Henkel, L. A., Johnson, M. K., \& De Leonardis, D. M. (1998). Aging and source monitoring: Cognitive processes and neuropsychological correlates. Journal of Experimental Psychology: General, 127, 251-268.

James, T. W., Humphrey, G. K., Gati, J. S., Menon, R. S., \& Goodale, M. A. (2000). The effects of visual object priming on brain activation before and after recognition. Current Biology, 10, 1017-1024.

Johnson, M. K. (2006). Memory and reality. American Psychologist, 61, $760-771$

Johnson, M. K., Foley, M. A., Suengas, A. G., \& Raye, C. L. (1988). Phenomenal characteristics of memories for perceived and imagined autobiographical events. Journal of Experimental Psychology: General, 117, 371-376.

Johnson, M. K., Hashtroudi, S., \& Lindsay, D. S. (1993). Source monitoring. Psychological Bulletin, 114, 3-28.

Johnson, M. R., \& Johnson, M. K. (2009). Top-down enhancement and suppression of activity in category-selective extrastriate cortex from an act of reflective attention. Journal of Cognitive Neuroscience, 21, 2320-2327.

Johnson, M. R., \& Johnson, M. K. (2014). Decoding individual natural scene representations during perception and imagery. Frontiers in Human Neuroscience, 8(59). doi:10.3389/fnhum.2014.00059

Johnson, J. D., McDuff, S. G. R., Rugg, M. D., \& Norman, K. A. (2009). Recollection, familiarity, and cortical reinstatement: A multivoxel pattern analysis. Neuron, 63, 697-708.

Johnson, M. K., \& Multhaup, K. S. (1992). Emotion and MEM. In S. Å. Christianson (Ed.), The handbook of emotion and memory: Research and theory (pp. 33-66). Hillsdale, NJ: Erlbaum.

Johnson, M. K., \& Raye, C. L. (1981). Reality monitoring. Psychological Review, 88, 67-85.

Karpel, M. E., Hoyer, W. J., \& Toglia, M. P. (2001). Accuracy and qualities of real and suggested memories: Nonspecific age differences. Journal of Gerontology: Psychological Sciences, 56B, 103-110.

Kensinger, E. A., Allard, E. R., \& Krendl, A. C. (2014). The effects of age on memory for socioemotional material: An affective neuroscience perspective. In P. Verhaeghen \& C. Hertzog (Eds.), The Oxford handbook of emotion, social cognition, and problem-solving in adulthood (pp. 26-46). New York: Oxford University Press.

Kim, H., \& Cabeza, R. (2007). Trusting our memories: Dissociating the neural correlates of confidence in veridical versus illusory memories. The Journal of Neuroscience, 27, 12190-12197.
Konkle, T., \& Oliva, A. (2012). A real-world size organization of object responses in occipitotemporal cortex. Neuron, 74, 1114-1124.

Koutstaal, W. (2003). Older adults encode — but do not always useperceptual details: Intentional versus unintentional effects of detail on memory judgments. Psychological Science, 14(2), 189-193.

Koutstaal, W., Reddy, C., Jackson, E. M., Prince, S., Cendan, D. L., \& Schacter, D. L. (2003). False recognition of abstract versus common objects in older and younger adults: Testing the semantic categorization account. Journal of Experimental Psychology: Learning, Memory, and Cognition, 29(4), 499-510.

Kuhl, B. A., \& Chun, M. M. (2014). Vivid remembering reactivates event-specific activity patterns in lateral parietal cortex and the hippocampus. Journal of Neuroscience, 34, 8051-8060.

Kuhl, B. A., Johnson, M. K., \& Chun, M. M. (2013). Dissociable neural mechanisms for goal-directed versus incidental memory reactivation. The Journal of Neuroscience, 33, 16099-16109.

Kuhl, B. A., Rissman, J., Chun, M. M., \& Wagner, A. D. (2011). Fidelity of neural reactivation reveals competition between memories. Proceedings of the National Academy of Sciences of the United States of America, 108, 5903-5908.

Kuhl, B. A., Rissman, J., \& Wagner, A. D. (2012). Multi-voxel patterns of visual category representation during episodic encoding are predictive of subsequent memory. Neuropsychologia, 50, 458-469.

Lee, S. H., Kravitz, D. J., \& Baker, C. I. (2012). Disentangling visual imagery and perception of real-world objects. NeuroImage, 59(4), $4064-4073$.

Levine, B., Svoboda, E., Hay, J. F., Winocur, G., \& Moscovitch, M. (2002). Aging and autobiographical memory: Dissociating episodic from semantic retrieval. Psychology and Aging, 17(4), 677-689.

Li, S.-C., Naveh-Benjamin, M., \& Lindenberger, U. (2005). Aging neuromodulation impairs associative binding: A neurocomputational account. Psychological Science, 16, 445-450.

Lieberman, M. D. (2006). Social cognitive neuroscience: A review of core processes. Annual Review of Psychology, 58, 259-289.

Ly, M., Murray, E., \& Yassa, M. A. (2013). Perceptual versus conceptual interference and pattern separation of verbal stimuli in young and older adults. Hippocampus, 23(6), 425-430.

Lyle, K. B., Bloise, S. M., \& Johnson, M. K. (2006). Age-related binding deficits and the content of false memories. Psychology and Aging, 21, 86-95.

May, C. P., Rahhal, T., Berry, E. M., \& Leighton, E. A. (2005). Aging, source memory, and emotion. Psychology and Aging, 20, 571-578.

McCabe, D. P., Roediger, H. L., III, McDaniel, M. A., \& Balota, D. A. (2009). Aging reduces veridical remembering but increases false remembering: Neuropsychological test correlates of rememberknow judgments. Neuropsychologia, 47(11), 2164-2173.

McDonough, I. M., Cervantes, S. N., Gray, S. J., \& Gallo, D. A. (2014). Memory's aging echo: Age-related decline in neural reactivation of perceptual details during recollection. NeuroImage, 98, 346-358.

McDonough, I. M., \& Gallo, D. A. (2013). Impaired retrieval monitoring for past and future autobiographical events in older adults. Psychology and Aging, 28, 457-466.

McDuff, S. G. R., Frankel, H. C., \& Norman, K. A. (2009). Multivoxel pattern analysis reveals increased memory targeting and reduced use of retrieved details during single-agenda source monitoring. Journal of Neuroscience, 29(2), 508-516.

McGinnis, D., \& Roberts, P. (1996). Qualitative characteristics of vivid memories attributed to real and imagined experiences. The American Journal of Psychology, 109, 59-77.

Miller, E. K., Freedman, D. J., \& Wallis, J. D. (2002). The prefrontal cortex: Categories, concepts and cognition. Philosophical Transactions of the Royal Society of London, 357, 1123-1136.

Mitchell, K. J., Ankudowich, E., Durbin, K. A., Greene, E. J., \& Johnson, M. K. (2013). Age-related differences in agenda-driven monitoring of format and task information. Neuropsychologia, 51, 2427-2441. 
Mitchell, K. J., \& Johnson, M. K. (2009). Source monitoring 15 years later: What have we learned from fMRI about the neural mechanisms of source memory? Psychological Bulletin, 135, 638-677.

Mitchell, K. J., Johnson, M. R., Higgins, J. A., \& Johnson, M. K. (2010). Age differences in brain activity during perceptual vs reflective attention. NeuroReport, 21, 293-297.

Mitchell, K. J., Johnson, M. K., Raye, C. L., Mather, M., \& D'Esposito, M. (2000). Aging and reflective processes of working memory: Binding and test load deficits. Psychology and Aging, 15, 527-541.

Mitchell, K. J., Johnson, M. K., \& Mather, M. (2003). Source monitoring and suggestibility to misinformation: Adult age-related differences. Applied Cognitive Psychology, 17, 107-119.

Mitchell, K. J., Raye, C. L., McGuire, J. T., Frankel, H., Greene, E. J., \& Johnson, M. K. (2008). Neuroimaging evidence for agendadependent monitoring of different features during short-term source memory tests. Journal of Experimental Psychology: Learning, Memory, and Cognition, 34, 780-790.

Naveh-Benjamin, M. (2000). Adult age differences in memory performance: Tests of an associative deficit hypothesis. Journal of Experimental Psychology: Learning, Memory, and Cognition, 26, 1170-1187.

Newman, S. D., Klatzky, R. L., Lederman, S. J., \& Just, M. A. (2005). Imagining material versus geometric properties of objects: An fMRI study. Cognitive Brain Research, 23, 235-246.

Norman, K. A., Polyn, S. M., Detre, G. J., \& Haxby, J. V. (2006). Beyond mind-reading: Multi-voxel pattern analysis of fMRI data. Trends in Cognitive Sciences, 10, 424-430.

Norman, K. A., \& Schacter, D. L. (1997). False recognition in younger and older adults: Exploring the characteristics of illusory memories. Memory \& Cognition, 25, 838-848.

Old, S. R., \& Naveh-Benjamin, M. (2008). Differential effects of age on item and associative measures of memory: A meta-analysis. Psychology and Aging, 23, 104-118.

Park, J., Carp, J., Hebrank, A., Park, D. C., \& Polk, T. A. (2010). Neural specificity predicts fluid processing ability in older adults. The Journal of Neuroscience, 30, 9253-9259.

Payer, D., Marshuetz, C., Sutton, B., Hebrank, A., Welsh, R. C., \& Park, D. C. (2006). Decreased neural specialization in old adults on a working memory task. NeuroReport, 17, 487-491.

Peelen, M. V., Atkinson, A. P., \& Vuilleumier, P. (2010). Supramodal representations of perceived emotions in the human brain. Journal of Neuroscience, 30, 10127-10134.

Polyn, S. M., Natu, V. S., Cohen, J. D., \& Norman, K. A. (2005). Category-specific cortical activity precedes retrieval during memory search. Science, 310, 1963-1966.

Rissman, J., \& Wagner, A. D. (2012). Distributed representations in memory: Insights from functional brain imaging. Annual Review of Psychology, 63, 101-128.

Rubin, D. C., \& Schulkind, M. D. (1997). Distribution of important and word-cued autobiographical memories in 20-, 35-, and 70-year-old adults. Psychology and Aging, 12, 524-535.
Scheibe, S., \& Carstensen, L. L. (2010). Emotional aging: Recent findings and future trends. Journal of Gerontology: Psychological Sciences, 65B(2), 135-144.

Schwarzlose, R. F., Swisher, J. D., Dang, S., \& Banisher, N. (2008). The distribution of category and location information across objectselective regions in human visual cortex. Proceedings of the National Academy of Sciences, 105, 4447-4452.

Shimamura, A. P. (2011). Episodic retrieval and the cortical binding of relational activity. Cognitive, Affective, \& Behavioral Neuroscience, 11(3), 277-291.

Simons, J. S., Owen, A. M., Fletcher, P. C., \& Burgess, P. W. (2005). Anterior prefrontal cortex and the recollection of contextual information. Neuropsychologia, 43(12), 1774-1783.

Simons, J. S., Peers, P. V., Mazuz, Y. S., Berryhill, M. E., \& Olson, I. R. (2010). Dissociation between memory accuracy and memory confidence following bilateral parietal lesions. Cerebral Cortex, 20, 479 485.

Spencer, W. D., \& Raz, N. (1995). Differential effects of aging on memory for content and context: A meta-analysis. Psychology and Aging, 10, 527-539.

Spreng, R. N., Wojtowicz, M., \& Grady, C. L. (2010). Reliable differences in brain activity between young and old adults: A quantitative meta-analysis across multiple cognitive domains. Neuroscience \& Biobehavioral Reviews, 34, 1178-1194.

Sreenivasan, K. K., Vytlacil, J., \& D’Esposito, M. D. (2014). Distributed and dynamic storage of working memory stimulus information in extrastriate cortex. Journal of Cognitive Neuroscience, 26, 11411153.

St-Laurent, M., Abdi, H., Bondad, A., \& Buchsbaum, B. R. (2014). Memory reactivation in healthy aging: Evidence of stimulusspecific dedifferentiation. The Journal of Neuroscience, 34(12), 4175-4186.

Tzourio-Mazoyer, N., Landeau, B., Papathanassiou, D., Crivello, F., Etard, O., Delcroix, N., \& Joliot, M. (2002). Automated anatomical labelling of activations in SPM using a macroscopic anatomical parcellation of the MNI MRI single-subject brain. NeuroImage, $15,273-289$

Vilberg, K. L., \& Rugg, M. D. (2008). Memory retrieval and the parietal cortex: A review of evidence from a dual-process perspective. Neuropsychologia, 46, 1787-1799.

Vingerhoets, G. (2014). Contribution of the posterior parietal cortex in reaching, grasping, and using objects and tools. Frontiers in Psychology, 5, 1-17.

Wagner, A. D., Shannon, B. J., Kahn, I., \& Buckner, R. L. (2005). Parietal lobe contributions to episodic memory retrieval. Trends in Cognitive Sciences, 9, 445-453.

Woods, R. P., Cherry, S. R., \& Mazziotta, J. C. (1992). Rapid automated algorithm for aligning and reslicing PET images. Journal of Computer Assisted Tomography, 16, 620-633. 